\title{
Interdisciplinary Approach for the Medical Management of Gestational Gigantomastia
}

\author{
Camila Cabrera, MD ${ }^{1}$ Mackenzy Radolec, MD ${ }^{1}$ Angela Prescott, MD ${ }^{2}$ Carolyn De La Cruz, MD ${ }^{2}$ \\ Stacy Beck, MD ${ }^{1}$ \\ 1 Department of Obstetrics and Gynecology, UPMC, Pittsburgh, \\ Pennsylvania \\ ${ }^{2}$ Department of Plastic Surgery, UPMC, Pittsburgh, Pennsylvania \\ Address for correspondence Camila Cabrera, MD, Department of \\ Obstetrics and Gynecology, UPMC, 300 Halket Street, Pittsburgh, \\ PA 15213-2582 (e-mail: cabrerac@upmc.edu).
}

Am J Perinatol Rep 2020;00:e304-e308.

Abstract
Keywords
- gigantomastia
- pregnancy
- cystic fibrosis
- infertility
- medical management
- interdisciplinary

Background Gestational gigantomastia is a rare and debilitating condition that is thought to result from hormone hypersensitivity. Several definitions have been proposed using breast weight and change in body mass index, but the breast growth is best summarized as rapid, diffuse, and excessive.

Case We report a case of a 31-year-old woman with a history of infertility and cystic fibrosis that developed pathologic breast growth during hormonal preparation for in vitro fertilization. Her serum laboratories were unremarkable, and she was medically managed until 31 weeks of gestation. After delivery, she experienced rapid decrease in breast size and was followed by plastic surgery with plan to allow spontaneous regression with interval breast reduction

Conclusion We highlight a successful interdisciplinary medical management approach, which helped to avoid a morbid, intrapartum breast reduction.

Gestational gigantomastia is a rare, unpredictable, and incapacitating disease that presents as rapid, diffuse, and excessive breast growth. Its cause is not well understood but is thought to be associated with hormone hypersensitivity and in some cases associated with autoimmune disorders. Medical complications include severe mastalgia, back pain, difficulty with ambulation, skin ulceration and necrosis, infections, and hemorrhages from exposed venous sinuses. ${ }^{1}$ Several reports have described severe cases necessitating pregnancy termination as well as mammoplasty and mastectomies that are commonly complicated by excessive blood loss and prolonged recoveries. ${ }^{2}$ Rare cases have even described sepsis, renal failure, and death. Psychosocial complications are also profound and include significant social anxiety. Due to the rarity and unpredictability of this disease, we present a case of interdisciplinary medical management that can potentially guide the management of future occurrences of this debilitating disease.

received

April 14, 2020

accepted

May 8, 2020
DOI https://doi.org/

$10.1055 / \mathrm{s}-0040-1715174$. ISSN 2157-6998.

\section{Case}

We present a case of a 31-year-old G1P0 with a history of primary infertility and cystic fibrosis. She initially presented to maternal fetal medicine (MFM) and genetics for preconception counseling in March of 2018. At that time, she was trying to conceive for a year and was initiating infertility work-up with reproductive endocrinology and infertility. At her initial visit, the patient was 5 feet 4 inches and her weight was 128 pounds for a body mass index of 22 .

At the start of her infertility work-up, the patient was using ovulation predictor kits and timed intercourse. Her work-up included hysterosalpingogram, which was normal, and a semen analysis, which was only significant for mild teratospermia (6-8\%). Pelvic ultrasound on February 23, 2018 showed an antral follicle count (AFC) of $30+$ with an arcuate uterus. Due to a high AFC and isolated episode anovulation as evidence by low cycle day 21 progesterone level, she was worked up for polycystic ovarian syndrome

Copyright $\odot 2020$ by Thieme Medical Publishers, Inc., 333 Seventh Avenue, New York, NY 10001, USA. Tel: $+1(212) 760-0888$.
License terms

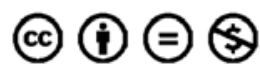


and her serum hormone levels were within normal limits. The patient did not have clinical signs of hyperandrogenism and otherwise was ovulating normally. She obtained clearance from cardiology and pulmonary medicine for pregnancy and ovulation induction protocol was started on March 22, 2018 with letrozole $2.5 \mathrm{mg}$ on cycle days 3 to 7 with cycle day 12 ultrasound, luteinizing hormone (LH), and progesterone levels. Patient had several cycles of ovulation induction and one cycle of intrauterine insemination, which failed. Between July 21, 2018 and August 21, 2018, the patient took combined oral contraceptive pills (COC) in preparation for in vitro fertilization (IVF) and noted an increase in breast size up to approximately a six-cup size in 2 to 4 weeks. In September 2018, she was started on medications to prepare for IVF egg retrieval, which included follicle stimulating hormone (FSH, Follistim), FSH combined with LH (Menopur), and gonadotropin-releasing hormone antagonist (Ganirelix). During this time, she did not note further growth in her breast size and her breasts were back to baseline within 3 to 5 weeks from the discontinuation of the COC. In October 2018, she began to use estradiol without any noticeable breast changes, but after November 14, 2018, days prior to the embryo transfer on November 18,2018 , she began to take progesterone supplementation and again noted a dramatic six-cup size increase in her breast size over 1 week. After the embryo transfer, she was transitioned to vaginal progesterone suppositories, but the breast growth continued.

The patient established prenatal care at 10 weeks of gestational age. At her 14-week visit, she complained of significant breast pain. A breast ultrasound showed no sonographic abnormalities and she was referred to Endocrinology. At 15 weeks (February 11, 2019) laboratories were obtained which included an intact parathyroid hormone $(\mathrm{PTH})<6.3$ (expected to be low in the first and second trimesters), Calcium 10.1 (normal), PTH related peptide 1.2 (normal), Vitamin D was normal, prolactin 112.5 (consistent with normal pregnancy levels), and thyroid stimulating hormone (TSH) 0.885 (normal for second trimester). She was started on bromocrip- tine $2.5 \mathrm{mg}$ twice daily and was increased to $5 \mathrm{mg}$ twice daily, which resulted in a slower rate of breast enlargement. In March, she was switched to cabergoline $0.5 \mathrm{mg}$, but the patient experienced accelerated rate of growth and in 1 week she was switched back to bromocriptine at a higher dose of $10 \mathrm{mg}$ twice daily. At 16 weeks, the patient met with surgical oncology (breast surgeon) and at 19 weeks she met with plastic surgery who reported she was not a surgical candidate due to the size, swelling, and increased vascularity of the breast. Physical therapy and manual lymphatic drainage (MLD) were recommended. At this time, the patient reported a 16-cup total increase in breast size, but the rate of growth had slowed (-Fig. 1). At 21 weeks, the patient developed superficial skin breakdown on the breast. Patient was seeing physical therapy during this time but with worsening ability to perform activities of daily living and worsening pain. Patient presented to labor and delivery triage at 23 weeks for uncontrolled pain and was encouraged to start oxycodone along with tylenol for pain control. She represented at 24 weeks with worsening pain and was admitted to the antepartum unit for pain control.

During her hospitalization, the patient's pain and discomfort progressed along with the growth of her breast tissue. Initially, oxycodone was up titrated to maximal doses, but ultimately she required physical medicine and rehab consultation for assistance with pain control. Patient was counseled thoroughly on neonatal abstinence syndrome and the likelihood of her own dependence on narcotic medications, which would require weaning. She was initiated on methadone and up titrated to a stable regimen of $30 \mathrm{mg}$ twice daily. Scheduled tylenol as well as oxycodone and hydromorphone for breakthrough pain during dressing changes allowed for stable and adequate pain control. Due to poor mobility related to pain, the patient was started on deep venous thrombosis prophylaxis with daily enoxaparin. Although the pain was better controlled, the other complications of both long-term hospitalization and skin breakdown and herniation of the breast tissue became problematic. At the time of admission, the patient had superficial wounds on her

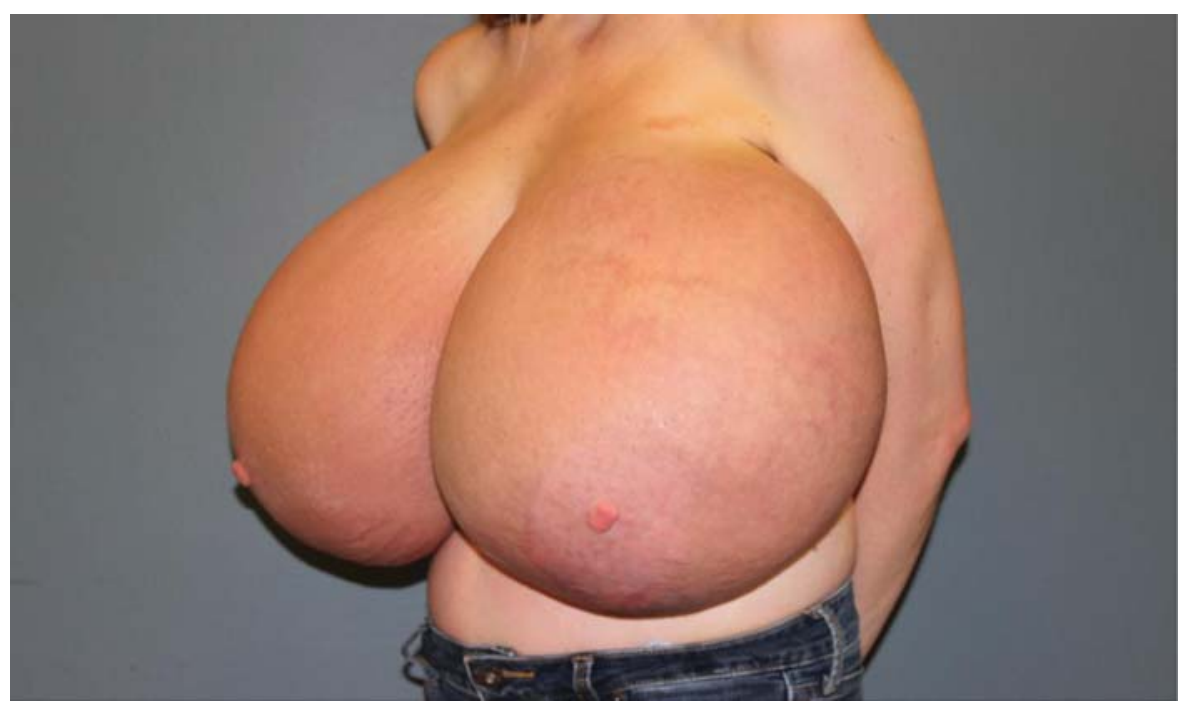

Fig. 1 Breast at 19 weeks of gestational age with peau d'orange appearance. 


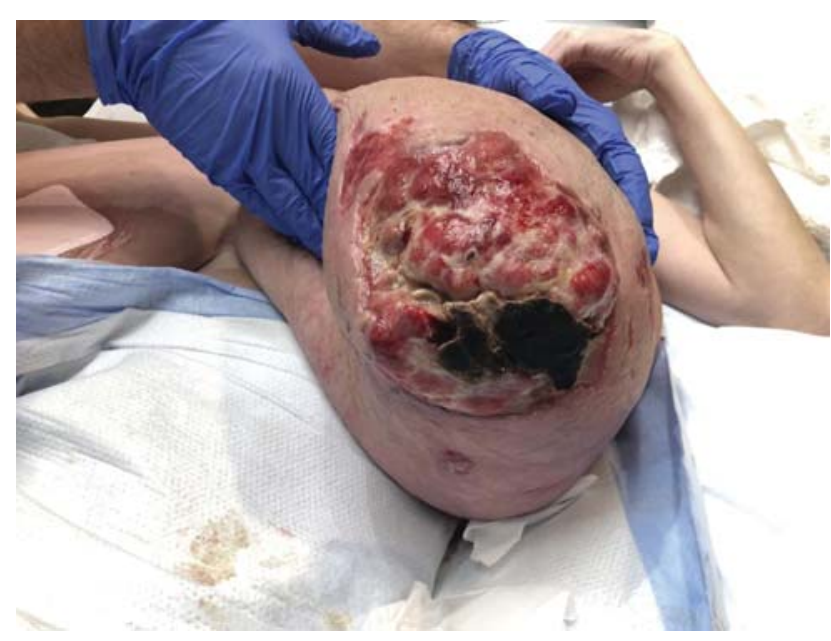

Fig. 2 Breast at 1 week of postpartum with evidence of herniation and exposure of venous sinus.

breast but as the breast grew, they ulcerated, herniated, and eventually hemorrhaged from exposed venous sinuses (-Fig. 2). She was seen by both surgical oncology (breast surgery) and plastic surgery during her admission. They again recommend avoidance of surgery during pregnancy due to concern for possible vascular compromise related to the large volume of blood contained in the growing breast. They did note worsening loss of skin integrity, growing wounds, and lymphovenous hypertension of the breast. Wound care routinely saw the patient and assisted in the management of her wounds. Green discoloration of her wound discharge was felt to be colonization with pseudomonas, but due to topical care with hypochlorous acid (Vashe) wet to dry dressings ( - Fig. 3 ), she remained without evidence of superimposed infection and never required antibiotics. For the lymphovenous hypertension, patient's mother and husband performed MLD as instructed by physical therapy. A compression garment was also made to measurement, which also helped significantly with her symptoms during her admission (-Fig. 4). Despite adequate wound care, venous sinus hemorrhages started to develop around

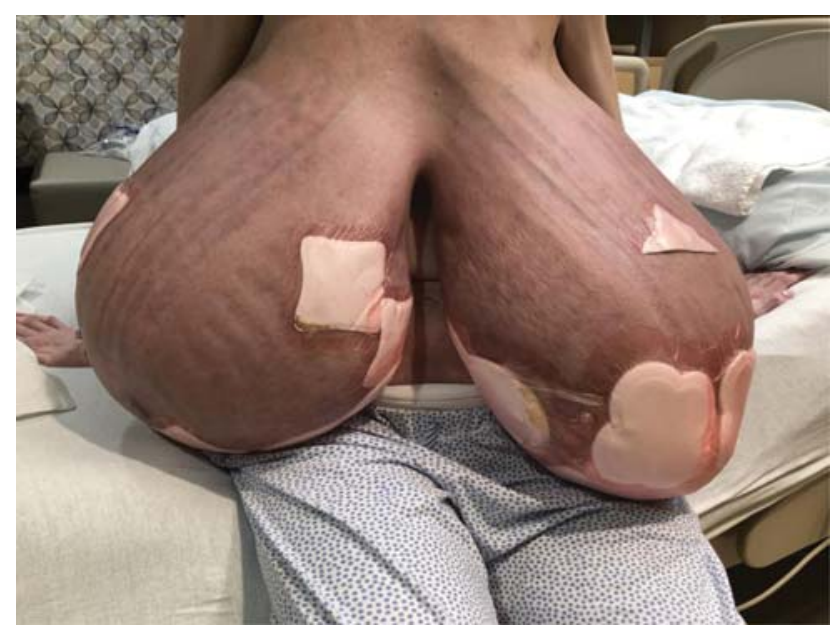

Fig. 3 Breast on postoperative day 5 with wet to dry dressings using Vashe hypochlorous acid.

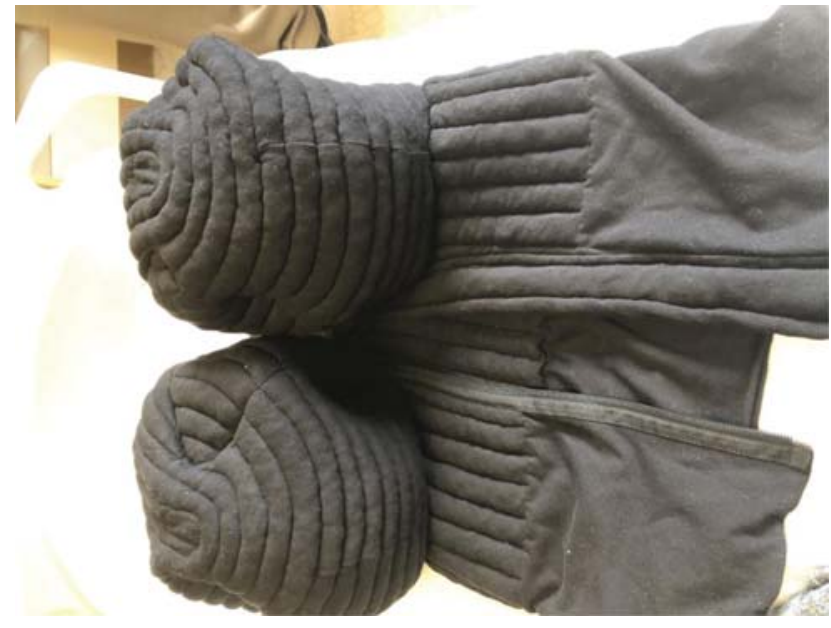

Fig. 4 Compression garment.

27 weeks. Anticoagulation was immediately discontinued, but she developed multiple episodes of hemorrhage (100-$300 \mathrm{cc}$ ) from exposed venous sinuses on her breast between 27 and 30 weeks. This was managed by suture ligation by plastic surgery. Due to this acute change in maternal status, the decision was made to administer betamethasone for fetal lung maturity. Bilateral IV access was established and patient was crossmatched for four units of packed red blood cells in anticipation for further breast hemorrhages. Her hemoglobin was optimized with the use of intravenous iron infusions. During this time, patient also developed visual hallucinations. Psychiatry was consulted for support and felt these symptoms were mediated by anxiety, panic, exhaustion, and delirium. She was started on venlafaxine, which was later switched to citalopram. Patient stabilized for a brief period but at 30 weeks developed recurrent breast hemorrhage and requested early delivery. After shared decision making with the patient and neonatology counseling on the risks of prematurity, decision was made for induction of labor at 31 weeks. She was moved to the labor suite at 30 weeks and 5 days for rescue dose of betamethasone as well as magnesium for fetal neuroprotection and underwent induction of labor at 31 weeks. The fetus developed recurrent late decelerations, which were unresponsive to resuscitative measures, and decision was made to proceed with primary Csection. The patient's neonate was appropriate for gestational age and remained in the neonatal intensive care unit for 6 weeks. The infant had respiratory distress syndrome with brief period of continuous positive airway pressure and stage II necrotizing enterocolitis that was treated with Zosyn for 1 week.

On postoperative day 5 , the patient noted her breasts felt softer and her compression garment was fitting loser. At this time, she began to record breast size (-Fig. 5). During her postpartum hospitalization, she was successfully weaned off methadone and pain was well controlled. Fortunately, she never developed pulmonary complications during her pregnancy or her recovery due to her cystic fibrosis or weight of the breasts. Once her breast wounds stabilized, she was deemed safe for discharge home with continued outpatient follow-up with 


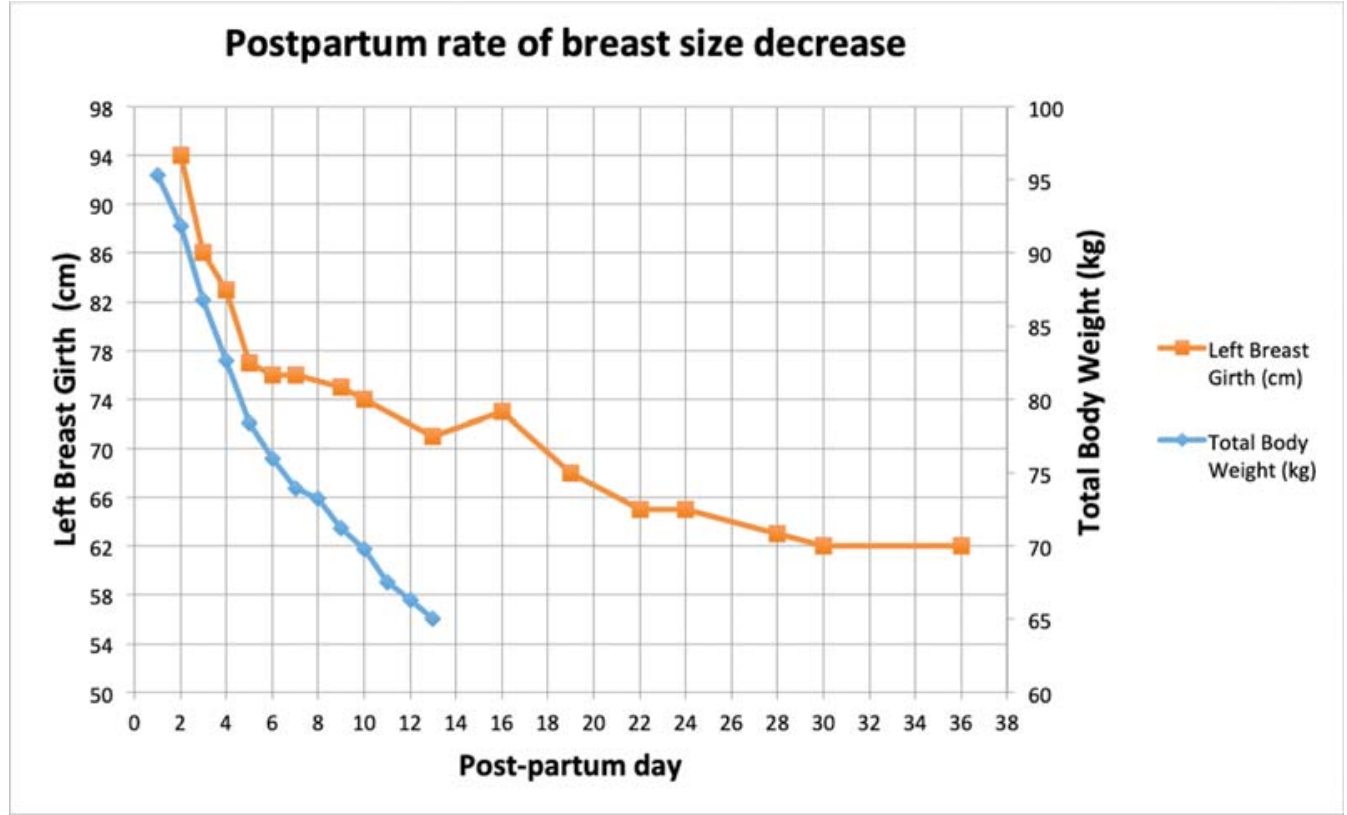

Fig. 5 Rate of breast size decrease in the postpartum period. Pre-pregnancy weight was $59 \mathrm{~kg}$.

wound care, plastic surgery, breast surgery, and MFM. At her 3-week postpartum visit, she was fully weaned off all narcotics without withdrawal symptoms. Plastic surgery recommended continued wound care to allow time for spontaneous regression with interval breast reduction. Patient did continue to struggle with significant anxiety and body image issues. At 2 months postpartum, the patient reported stabilization of her breast size and the plan was made for breast reduction with free nipple grafts, which was performed 20 weeks postpartum ( - Fig. 6). At time of surgery, total 4,320 and 4,762 g of breast tissue were removed from the left and right breast, respectively. Estimated blood loss for the procedure was 500cc. Patient was discharged on postoperative day 1 with bilateral Jackson-Pratt drains in place.

\section{Discussion}

The incidence of gestational gigantomastia is quoted to be between 1 in 28,000 and 1 in 118,000 deliveries. ${ }^{3}$ The etiology is not understood, but cases have been seen in association with medications, autoimmune disorders, hormone receptor hypersensitivity, and concurrent malignancy. ${ }^{1}$ Risk factors are also not well understood, but it has been most commonly reported in Caucasian and multiparous women. The majority of literature is composed of case reports and small case series. ${ }^{2}$ The majority of case reports focus on surgical management of this complex disease. We would like to highlight the value of supportive and medical management, which should include the use of an

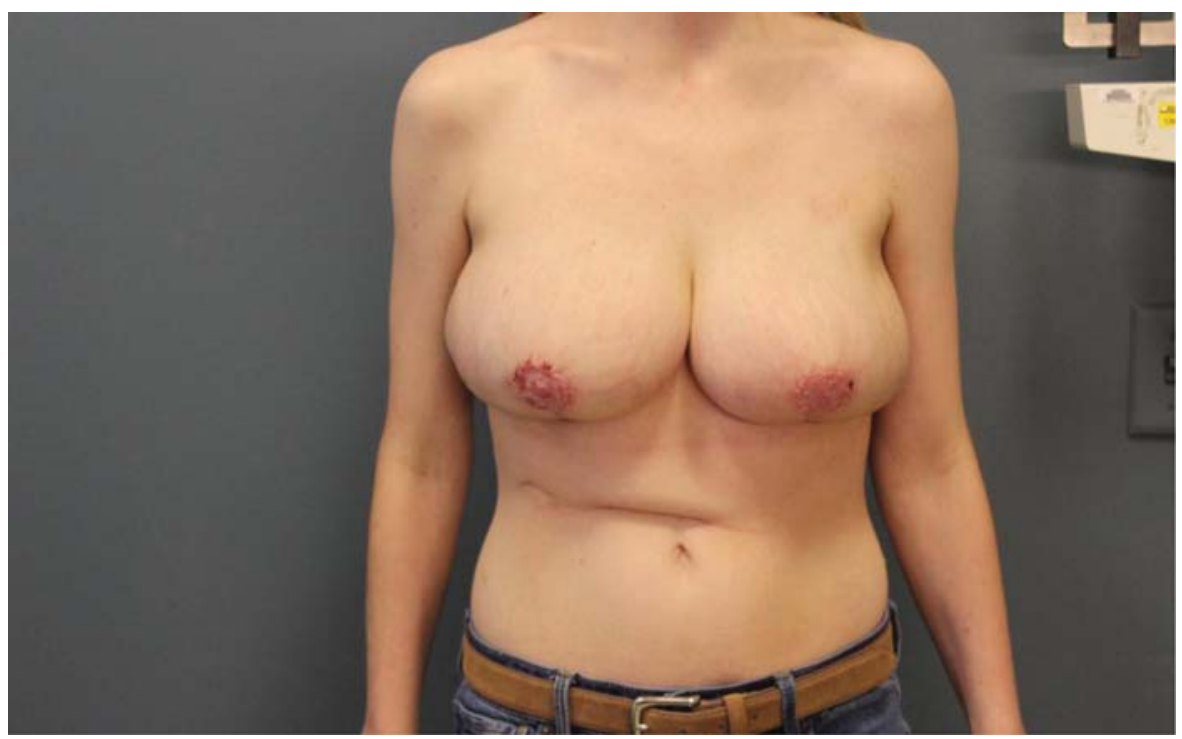

Fig. 6 Postoperative picture after bilateral breast reduction with free nipple graft which was done 20 weeks of postpartum. 
interdisciplinary team including MFM, plastic surgery, endocrinology, physical medicine and rehabilitation for pain management, wound care, physical therapy with MLD, and support garments and psychiatry as needed. From the patient's perspective, the bromocriptine, MLD, and the custom compression garment were felt to be helpful for her symptoms. A literature review of 23 cases that have been reported since 1968 created an algorithm for management of gestational gigantomastia. This algorithm states that if maternal disability and fetal compromise allow, supportive management can be implemented. It also indicates that reduction mammoplasty is preferred in women who do not desire future fertility as the risk of recurrence is significantly higher than in those who undergo bilateral mastectomies with reconstruction. ${ }^{3}$ This patient did not desire future fertility therefore breast reduction was the appropriate surgery for her. In many of the cases reported, similar to our case, the typical response to bromocriptine was slowed rate of breast growth, but regression of breast size was not observed. ${ }^{4}$ None of the cases we reviewed discuss the use of MLD, or the use of a support garment as adjunct methods of supportive management. Our patient's temporal relation of rapid breast growth with the use of COC pre-pregnancy, regression to baseline several weeks after discontinuation, and then recurrent rapid increase in size with progesterone used during IVF pregnancy supports the theory that this disease is at least in part hormone mediated. In some gigantomastia case reports, non-PTH related hypercalcemia is described (possibly secondary to high parathyroid hormone-related protein secretion from breast tissue). This phenomenon was not seen in our patient as all her serum laboratories were within normal limits of pregnancy. Our patient's breast size stabilized, and she has now undergone breast reduction by plastic surgery.

Conflict of Interest

None declared.

\section{References}

1 Rezai S, Nakagawa JT, Tedesco J, et al. Gestational gigantomastia complicating pregnancy: a case report and review of the literature. Case Rep Obstet Gynecol 2015;2015:892369

2 Mangla M, Singla D. Gestational gigantomastia: a systematic review of case reports. J Midlife Health 2017;8(01):40-44

3 Swelstad MR, Swelstad BB, Rao VK, Gutowski KA. Management of gestational gigantomastia. Plast Reconstr Surg 2006;118(04): 840-848

4 Hedberg K, Karlsson K, Lindstedt G. Gigantomastia during pregnancy: effect of a dopamine agonist. Am J Obstet Gynecol 1979; 133(08):928-931 